Gut, 1980, 21, 18-22

\title{
Thiocyanate as a marker of saliva in gastric juice ?
}

\author{
P B BOUlos, P F Whitfield, M DAVE, R G FABER, AND M HOBSLEY \\ From the Department of Surgical Studies, The Middlesex Hospital and Medical School, London
}

SUMMARY One source of error in gastric secretion studies is swallowed saliva. The possibility that salivary thiocyanate might be used to measure this contamination has been investigated. Thiocyanate concentration was measured in saliva and gastric juice collected simultaneously in 22 duodenal ulcer patients undergoing routine insulin and histamine secretion studies. On stimulation, despite the increase in the rate of gastric secretion this was not matched by an appropriate fall in the concentration of thiocyanate in gastric juice. Moreover, in one-third of the gastric juice specimens, the thiocyanate concentration was greater than in the simultaneous samples of saliva. Thus, contrary to what has been claimed, thiocyanate is present not only in saliva but also in gastric juice. Therefore it cannot be used as a marker of salivary contamination. An adequate marker of this source of error has not yet been found.

When gastric secretion studies are performed, errors due to duodenogastric reflux, swallowed saliva, and loss of gastric juice through the pylorus are usually ignored. Instillation of phenol red into the stomach as a dilution indicator (Hobsley and Silen, 1969) has been used to measure pyloric losses. The volume of duodenogastric reflux in a sample of gastric juice can be calculated using a formula based on the output of sodium ions (Hobsley, 1974). The accuracy of quantitative analysis is improved when such errors are eliminated (Hassan and Hobsley, 1971).

Many workers have attempted to prevent swallowing of saliva by asking their patients to expectorate saliva (Kirsner et al., 1956; Baron, 1963) or by the insertion of dental plugs which were repeatedly changed (Makhlouf et al., 1966) or by means of continuous suction (Ihre, 1938). These methods themselves increase salivary flow and with none of them can it be certain that salivary contamination has been prevented.

If one accepts that it is impossible to prevent swallowing of saliva, correction for errors caused by its presence can alternatively be made by calculating the volume of contamination in the aspirated gastric juice. Teichmann (1953) claimed that thiocyanate is found only in saliva and its presence in the gastric juice indicates probable contamination. If this were

${ }^{1}$ Based on a paper read at the British Society of Gastroenterology, York, 1977.

Received for publication 8 August 1979. true, then, by using thiocyanate as a marker and measuring its concentration in the saliva and gastric juice, the volume of swallowed saliva could be determined. Additional studies were also performed to determine whether thiocyanate was present in duodenal contents.

\section{Methods}

PATIENTS

Studies were conducted in 22 duodenal ulcer patients undergoing routine gastric secretion studies, 19 of whom had had vagotomy. Each patient fasted from midnight and at 9.00 am the patient lay semirecumbent on a couch. A special two-lumen nasogastric tube was passed (Thomson et al., 1973) and the position checked by the water-recovery test of Hassan and Hobsley (1970). In all subjects phenol red was instilled into the stomach by the small lumen. After a basal collection of half an hour, soluble insulin 0.2 units $/ \mathrm{kg}$ was injected intravenously and collections made for two hours, then histamine acid phosphate was infused intravenously for one hour at the rate of $0.13 \mathrm{nmol} \mathrm{kg}^{-1}$ hour-1 using a Palmer infusion pump (Lawrie et al., 1964). Blood sugar was measured and only tests where hypoglycaemia of less than $2.2 \mathrm{mmol} / 1$ was achieved were included.

Disposable dental suckers were placed under the tongue and continuous suction maintained at a negative pressure of $10 \mathrm{mmHg}$ using a Robert's 
pump; each small volume of saliva was collected in a test tube placed within the flask of the pump. Gastric juice was collected using an automatic gastric suction pump (United Analysts Ltd.). Specimens of saliva and gastric juice were collected simultaneously and, when the volume was inadequate for analysis, consecutive specimens were pooled. In some patients no samples of saliva could be obtained in one or other of the periods.

Pancreatic juice was collected during endoscopic intubation of the pancreatic duct in six patients and analysed for thiocyanate content. Bile was collected during cholecystectomy in four patients and a similar analysis attempted.

The volume of each sample of saliva and gastric juice was measured and recorded. The gastric juice was filtered through Whatman No. 1 filter paper and the following measurements were made: titratable acidity (Radiometer automatic titrator, titration to $\mathrm{pH} 7$ ), sodium, potassium, and chloride concentrations (EEL flame photometer and chloride meter), and phenol red concentration (Unicam SP spectrophotometer) as described by Faber et al. (1975). The observed volume of each 10-minute sample after correction for pyloric losses by means of the marker phenol red (Hobsley and Silen, 1969) was designated $\mathrm{V}_{\text {corr }} \mathrm{ml} / 10 \mathrm{~min}$. The volume rate of secretion during the pre-stimulation period was expressed as the mean value for 10 minutes; during insulin, stimulation was expressed as the mean value for 10 minutes during the period $\frac{1}{2}-2$ hours after the injection of insulin; and during histamine stimulation was expressed as the mean value per 10 minutes for all samples after the first 10-minute sample.
Thiocyanate in the samples of saliva and gastric juice was measured using pyridine-cupric sulphate reagent (Vogel, 1953).

Recovery experiments using known quantities of thiocyanate added to samples of gastric juice containing phenol red gave a correspondence between theoretical and observed values to within 0.02 $\mathrm{mmol} / \mathrm{l}$ of thiocyanate.

The mean of the volumes and concentration for each period in each study were calculated and differences were tested using the Wilcoxon paired rank sum test.

\section{Results}

In 22 patients, 198 paired, 10-minute samples of saliva and gastric juice were collected simultaneously. The concentrations of thiocyanate in the gastric juice [CNS] and in the saliva [CNS]s are plotted in Fig. 1. The diagonal line represents the equality between the two samples of each pair. In other words, any point lying to the right and below the line was one in which the concentration of thiocyanate was greater in the saliva than in the gastric juice. On the other hand, any point lying on the left and above the line was one in which the concentration of thiocyanate was greater in the gastric juice than in saliva. The latter situation was obtained in 60 of the sample-pairs.

The average volume rates of secretion during pre-stimulation, insulin and histamine periods are shown in Fig. 2. The insulin-stimulated secretion in these preponderantly post-vagotomy subjects was not significantly greater than the pre-stimulation

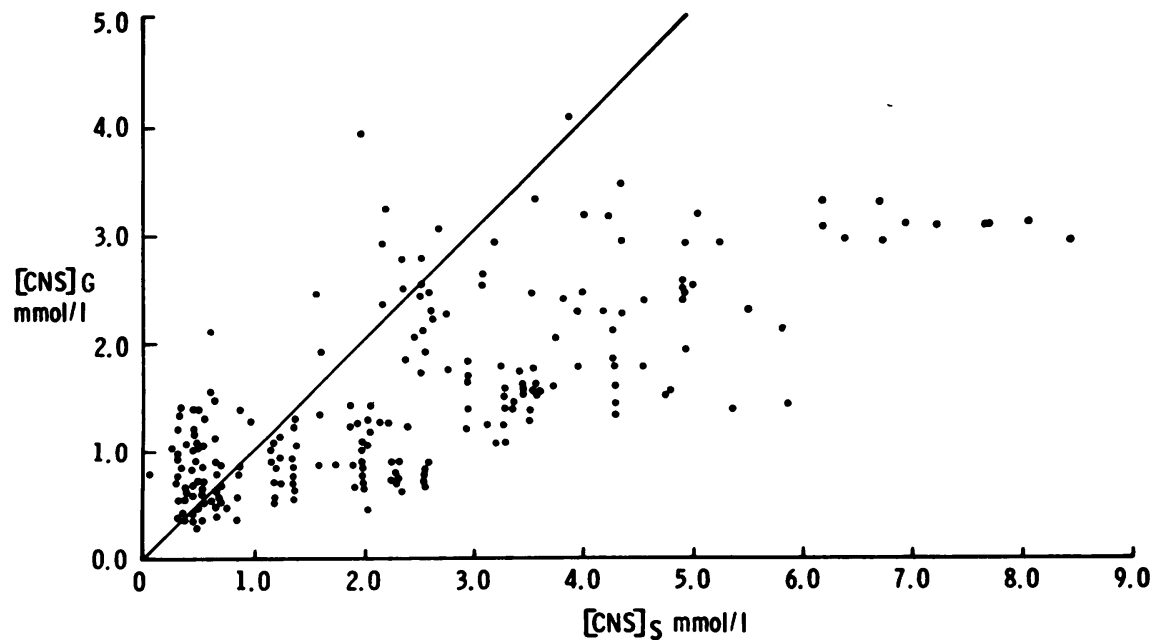

Fig. 1 Concentrations of thiocyanate in gastric juice plotted against thiocyanate concentrations in saliva. The diagonal line represents equality between two samples of each pair. 


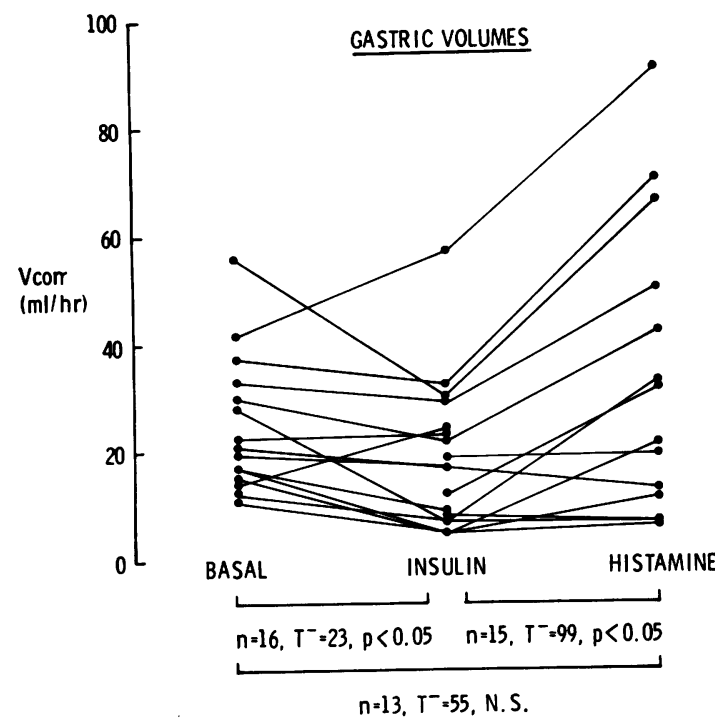

Fig. 2 Volumes of gastric secretion before and after stimulation with insulin and with histamine.

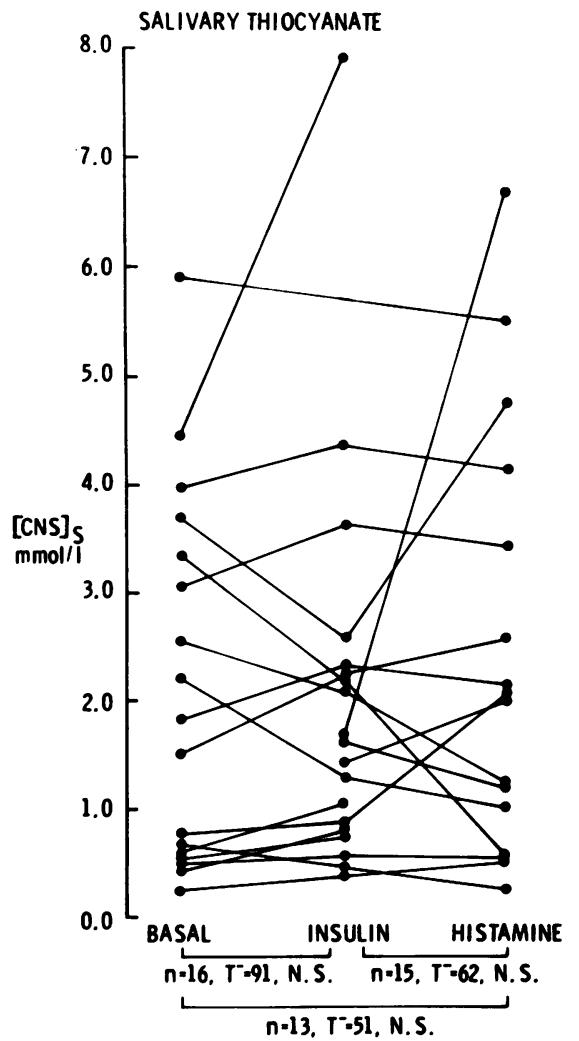

secretion; indeed, it was marginally less $(T=23$; $P \cong 0.05)$. However, the histamine-stimulated secretion was definitely greater than the insulin-stimulated secretion $(T=99 ; P<0.05)$ and the median percentage increase was $76 \%$. The concentrations of thiocyanate in the saliva and gastric juice during prestimulation and stimulated periods are shown in Fig. 3. Stimulation by insulin did not cause any significant change in the thiocyanate concentration of either saliva $(T=91)$ or gastric juice $(T=65 \cdot 5)$. Stimulation by histamine produced no change in salivary thiocyanate concentration $(T=62)$; it did produce a reduction in gastric juice thiocyanate concentration $(T=23)$, but the magnitude of this change was small; the median percentage decrease was $14 \%$-that is, about one-sixth of the corresponding rise in volume of secretion.

In the six specimens of pancreatic juice, no thiocyanate was found. It was impossible, with the method being used, to measure thiocyanate in bile because of the turbidity of bile.

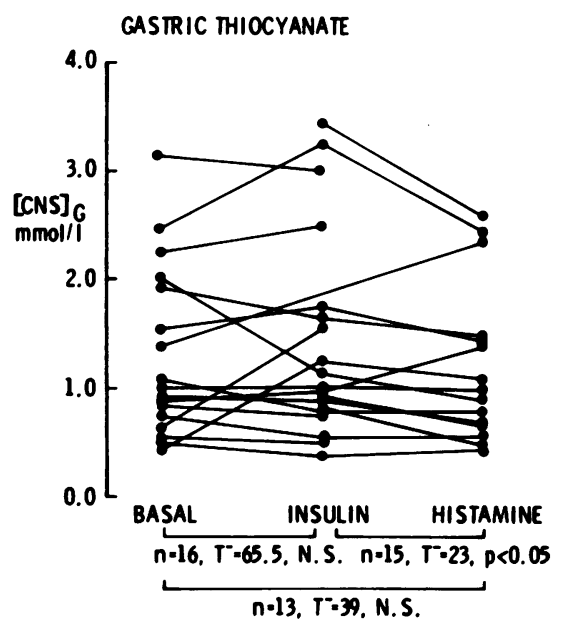

Fig. 3 Concentrations of thiocyanate in the saliva and gastric juice before and after stimulation with insulin and with histamine. 


\section{Discussion}

Thiocyanate present in body fluids is derived from alimentary absorption of preformed thiocyanate from food such as milk and vegetables and from detoxication of cyanide (Støa, 1957). It is a potent catalyst of nitrosation (Boyland and Walker, 1974) of secondary amines with nitrite to produce nitrosamines, a reaction which has been shown to occur in vitro in human gastric juice (Sen et al., 1969). Since Teichmann (1953) suggested that thiocyanate in the gastric juice is contamination from swallowed saliva, there have been no studies to clarify his statement until Ruddell et al. (1977), studying thiocyanate in the fasting and secreting stomach and in saliva, also concluded that thiocyanate in gastric juice was of a salivary origin. We have therefore attempted to obtain further evidence that one can use thiocyanate as a marker in order to measure the volume of saliva swallowed in the gastric juice. It has been shown that thiocyanate levels in the gastric juice and in saliva is higher in smokers than in nonsmokers because of absorption and metabolism of cyanide compounds in tobacco smoke (Boyland and Walker, 1974). We have eliminated this variable factor by studying only patients who smoked more than 15 cigarettes daily.

The hypothesis that there is no thiocyanate in the gastric juice leads to the following deductions: (1) thiocyanate concentrations in gastric juice should always be lower than in simultaneously collected saliva; (2) when gastric secretion is stimulated and aspirated, gastric juice therefore contains a lower proportion of swallowed saliva, and the thiocyanate concentration in the aspirated juice should fall appropriately.

Our results contradict these two deductions. Firstly, one-third of the gastric juice samples had a higher thiocyanate concentration than the corresponding salivary sample; secondly, the fall in gastric juice thiocyanate concentration was inappropriately small compared with the rise in volume during histamine stimulation. These results strongly indicate that, in at least some samples of gastric juice, not all the thiocyanate can originate in the saliva.

The thiocyanate in aspirated juice, if it does not originate in saliva, might arise in secretion from the stomach or refluxed fluid in the duodenum. Although it was not the aim of this study to find out this extra salivary source of thiocyanate our studies did not detect thiocyanate in pure pancreatic juice and so that possibility has been excluded.

It is difficult to explain the differences between our results and those of Ruddell et al. (1977). Their method of measurement of thiocyanate (Johnson, 1916) was different from ours and their ranges of values for thiocyanate concentration in saliva and in aspirated stomach contents were much lower than ours. Moreover, they did not study paired values for thiocyanate in gastric aspirate and in simultaneously obtained saliva. They reported a fall in thiocyanate concentration in gastric aspirate during stimulation of gastric secretory output, but it is noteworthy that their graph shows no corresponding increase in thiocyanate concentration when the gastric secretion rate fell towards normal after the period of stimulation had ended; thiocyanate concentration remained unchanged.

Clearly, additional work is necessary to resolve the discrepancies in the available data. However, from the data of our own studies we conclude that thiocyanate at present cannot be relied upon as a marker of saliva in aspirated gastric juice.

\section{References}

Baron, J. H. (1963). Studies of basal and peak acid output with an augmented histamine test. Gut, 4, 136-144.

Boyland, E., and Walker, S. A. (1974). Effect of thiocyanate on nitrosation of amines. Nature, 248, 601-602.

Faber, R. G., Russell, R. C. G., Parkin, J. V., Whitfield, P., and Hobsley, M. (1975). The predictive accuracy of the post-vagotomy insulin test: a new interpretation. Gut, 16, 337-342.

Hassan, M. A., and Hobsley, M. (1970). Positioning of subject and of nasogastric tube during a gastric secretion study. British Medical Journal, 1, 458-460.

Hassan, M. A., and Hobsley, M. (1971). The accurate assessment of maximal gastric secretion in control subjects and patients with duodenal ulcer. British Journal of Surgery, 58, 171-179.

Hobsley, M. (1974). Pyloric reflux: a modification of the two component hypothesis of gastric secretion. Clinical Science and Molecular Medicine, 47, 131-141.

Hobsley, M., and Silen, W. (1969). Use of an inert marker (phenol red) to improve accuracy in gastric secretion studies. Gut, 10, 787-795.

Ihre, B. (1938). Human gastric secretion. Acta Medica Scandinavica, suppl. 95.

Johnson, M. O. (1916). On the determination of small quantities of hydrocyanic acid. Journal of the American Chemical Society, 38, 1230-1235.

Kirsner, J. B., Bock, D., Palmer, W. L., Levin, E., and Ford, H. (1965). Variations in basal gastric secretion in man and the evaluation of gastric secretory stimulus. Gastroenterology, 30, 779-789.

Lawrie, J. H., Smith, G. M. R., and Forrest, A. P. M. (1964). The histamine-infusion test. Lancet, 2, 270-273.

Makhlouf, G. M., McManus, J. P. A., and Card, W. I. (1966). A quantitative statement of the two-component hypothesis of gastric secretion. Gastroentero$\operatorname{logy}, 51,149-171$.

Ruddell, W. S. J., Blendis, L. M., and Walters, C. L. (1977). Nitrite and thiocyanate in the fasting and secreting stomach and in saliva. Gut, 18, 73-77.

Sen, N. P., Smith, D. C., and Schwinghamer, L. (1969). 
Formation of $\mathrm{N}$-nitrosamines from secondary amines and nitrite in human and animal gastric juice. Food and Cosmetics Toxicology, 7, 301-307.

Støa, K. F. (1957). Studies on thiocyanate in serum with some supplementary investigations in saliva, urine, and cerebrospinal fluid. Universitat $i$ Bergen Arbok, Medisinsk Rekke, 2.

Teichmann, W. (1953). Untersuchungen über den Gehalt des menschlichen magensaftes an Eiweisskörbern und das Vorkommen von Imidazolkörpern. Deutsche Zeitschrift für Verdauungs- und Stoffwechselkrankheiten, 13, 203-211.

Thomson, J. P. S., Russell, R. C. G., and Hobsley, M. (1973). Double lumen tube for gastric secretion studies. British Medical Journal, 3, 175.

Vogel, A. I. (1953). A Text-book of Macro and Semimicroqualitative Inorganic Analysis, p. 347. Longmans: London. 